\title{
The comparing of acute effects of two training models aerobic and resistance on the clotting times in young women students
}

\author{
Rahmani Ghobadi M. ${ }^{1 \mathrm{ABCDE}}$, Forghani Ozrudi M. ${ }^{2 \mathrm{ABCDE}}$ \\ ${ }^{1}$ Department of Physical Education \& Sport Sciences, Damavand Branch, Islamic Azad University, Damavand, Iran \\ ${ }^{2}$ Young Researchers and Elite Club, Babol Branch, Islamic Azad University, Babol, Iran
}

Authors' Contribution: A - Study design; B - Data collection; C - Statistical analysis; D - Manuscript Preparation; E-Funds Collection.

\begin{abstract}
Purpose: $\quad$ Some of the benefits of physical activity may result from effects on hemostasis. However, the increased burden of cardiovascular complications and sudden death occurring during and immediately after exercise prompts investigation to elucidate the biological relationship between physical exercise and hemostatic function. The aim of this study was to determine effects of one bout exhaustive aerobic exercise and resistance training on the clotting times in healthy young women student.

Material: $\quad$ Thirty trained volunteer female students of physical education were selected objectively and availability. The subjects were randomly divided into two groups of aerobic $(n=15)$ and resistance training $(n=15)$. Aerobic group performed exhaustive workout program on treadmill intensity 65 to $75 \% \mathrm{VO}_{2 \max }$ on treadmill. The resistance group completed three sets of 5-7 repetitions of six exercises at an intensity corresponding to $80 \%$ of 1 RM. Following 12 to 14 hours of nightly fasting, venous blood samples ( $5 \mathrm{cc}$ ) were collected pre, immediately after exercise and after 60 min of recovery and analyzed for PT (Prothrombin Time) and aPTT (Activated Partial Thromboplastin Time) times. Participants were matched according to anthropometric measurements, age and $\mathrm{VO}_{2 \max }$. Hypothesizes were tested by using independent $\mathrm{t}$, repeated measures and post-hoc test ( $\mathrm{p} \leq 0.05)$.

Results: $\quad$ One bout of the exhaustive aerobic exercise and resistance training caused significantly shortening in PT $(p<0.001)$ and aPTT $(p=0.006, p<0.001$ respectively) times at immediately after exercise. Also aPTT $(p=0.005$, $p<0.001$ respectively) times significantly increased after recovery in comparison with baseline levels in two groups. No significantly differences observed in PT and aPTT between two groups in all stages. The results show that exhaustive aerobic exercise and resistance training lead to mild and transit increase in coagulation system and induced shortening clotting times. Exercise-related hypercoagulability is mainly due to an increase in coagulation factor VIII (FVIII) with no parallel alterations of other clotting factors.

Conclusions: Similar to endurance exercise, FVIII activity raises following resistance exercise, and appears to be positively correlated with the volume of weight lifted. The ß-adrenergic pathway has been implicated as a possible pathway mediating an exercise- induced increase in FVIII and decease clotting time.

Keywords: clotting time, exercise training, PT, aPTT, young women students, blood, coagulation factor.
\end{abstract}

\section{Introduction}

Blood coagulation homeostasis system is an important mechanism that involves a complex set of interactions between protease enzymes and factors that lead to the production of thrombin and fibrin clots are rich. The mechanisms and homeostasis are involved in the regulation of different routes, each of which may be controlled by different regulatory factors [1]. Based on the results, safe and effective exercise of moderate intensity cardiovascular disease can be reduced. A moderate-intensity exercise 50 to $74 \%$ of maximum oxygen consumption, the suppression of platelet reactivity and development Fibrinolytics, will not lead to changes in the coagulation system. However, acute exercise intensity more than $74 \%$ of maximum oxygen consumption simultaneously Fibrinolyticy activity, platelet coagulation and develop their reactivity [2]. Are some of the benefits of physical activity may be due to its effects on hemostasis system. But increased cardiovascular complications and sudden death occurring during or immediately after exercise, further investigation to find the connection between exercise and performance necessary hemostasis [3, 4]. Prothrombin time (1appT) (c) Rahmani Ghobadi M., Forghani Ozrudi M., 2019 doi:10.15561/20755279.2019.0406 external and internal indicators of the blood coagulation and the changes these factors remain in the body after 1 to 24 hours [1]. Menzel and Hilberg Effect of a session of aerobic exercise aerobic threshold of $80 \%$ of the young and middle-aged men, a significant decrease in time aPPT (respectively 8\% and 6\%) and PT showed no significant changes [5]. Based on the results Lekakis et al a session of aerobic exercise in patients with hypertension was associated with a significant reduction apt and increase PT [6]. Also Hilberg et al (2003) reported that 60 to 120 minutes of aerobic exercise with less than $90 \%$ lactate threshold intensity leads to significant shortening APPT immediately (14.8\%) and 2 hours (9.7\%) after practice and unchanged of PT immediately and significantly decreased in 2 hours after exercise (relative to baseline) it [7]. Unlike aerobic exercise on resistance exercise, increased physical pressure with minimal mobility. Research shows that even low-intensity resistance training, when practiced with restricted blood flow medium flame, they can effectively induce Hypertrophy and increase their power. Women coagulation response after a bout of resistance exercise has been shown that young women with the percentage of fat may be at risk of harmful clots forming 
at rest and during exercise than women to be skinny [8]. In recent years, resistance training, part of sports athletes and regular exercisers form. The exercises are widely used to improve physical fitness \& also increase muscle size, injuries are prevented. Therefore, to increase the power, capacity and rehabilitation of elderly patients are recommended. It is also possible exercises for recreation and acute in many cases by people with varied fitness. The knowledge and insights about the impact of this practice on the various systems of the body including blood clotting seems essential.

The purpose of this study is acute effects of resistance training and aerobic submaximal a meeting on young women students clotting times were determined and compared. The researchers are looking for that resistance and aerobic training affect PT \& aPTT in young women students?

\section{Materials and Methods \\ Participants}

This quasi-experimental study was conducted on healthy women student and young active and smoking and hormones, as was Voluntary. After medical examinations and ensure the health and beam testing, 20 healthy volunteers and active with an average age of 19.12 \pm 1.09 (years), height $163.56 \pm 4.64(\mathrm{~cm})$, weight $58.31 \pm 7.59(\mathrm{~kg})$ $\mathrm{VO}_{2 \max } 36.94 \pm 4.61$ (min. $\mathrm{kg} / \mathrm{mL}$ ) and $22.18 \pm 3.67 \%$ fat selected randomly in two groups: resistance training and aerobic exercise groups. Once familiar with the exercise protocol, subjects completed consent form.

\section{Research Design}

Aerobic exercises include a session on the treadmill running with intensity $\mathrm{VO}_{2 \max } 65 \%-75 \%$ was to exhaustion. Treadmill speed gradually and beat until the subject's heart rate reserve (Karvonen) increased intensity during practice and was maintained until exhaustion. Control Polar heart rate monitor was used during the activity. Group strength training protocol consists of 10 minutes of warm-up stretching, weight training six major muscles of the upper and lower extremities (leg press, bent and open, the bench press, pull armpit and elbow flexion) in three sets, 5 to 7 repeat and with $80 \%$ of one repetition maximum (1RM) with 1-minute rest between sets. Abnormal symptoms in a person, no change in heart rate, reach $90 \%$ heart rate forecast and announced the discontinuation of the practice test were considered by the subjects as well as a stop [15]. Blood samples in three stages (after a 30-minute break), immediately and 60 minutes after the completion of the exercise of venous antibiotics cubital and after 12 to 14 hours of fasting night between the hours of 7 to 9 am (in the control of circadian rhythms body) was performed. PT and aPTT times using Laboratory kits Diagnostica French and Biomerieux Coagulometer device was measured.

\section{Statistical Analysis}

For analyzing the data, we used Kolmogorov-Smirnov Test for checking data normality, hypothesizes were tested by using independent $t$, repeated measures and post-hoc test $(\mathrm{p} \leq 0.05)$, by SPSS22 software.

\section{Results}

Anthropometric parameters including weight, body fat percentage and maximum oxygen consumption of the samples showed no significant changes. Immediately after the exercise, aerobic and resistance training groups, PT values, respectively, 0.42 and 0.44 seconds was significantly decreased $(\mathrm{p}<0.01)$ and 60 minutes after the end of practice, the only significant increase in the resistance group training compared to the base case $(p<0.001)$. aPTT levels of aerobic and resistance groups, respectively, 0.54 and 1.33 seconds, reduced $(p<0.001$, $\mathrm{p}=0.006$ ), respectively, and 60 minutes after exercise to levels significantly increased over baseline $(p<0.001$, $\mathrm{p}=0.005$ ), respectively. Between the two groups at different stages of apt and PT tests the difference was not significant.

Table 1. Mean and standard deviance aPPT and PT of groups

\begin{tabular}{|c|c|c|c|}
\hline \multirow[b]{2}{*}{ Process } & \multicolumn{3}{|l|}{ aPTT } \\
\hline & $\begin{array}{l}\text { Before the } \\
\text { test }\end{array}$ & Immediately & $\begin{array}{l}1 \mathrm{~h} \text { after the } \\
\text { test }\end{array}$ \\
\hline $\begin{array}{l}\text { resistance } \\
\text { training }\end{array}$ & $38.31 \pm 1.43$ & $36.98 \pm 1.55 a$ & $39.00 \pm 1.41 b$ \\
\hline \multirow[t]{3}{*}{ Aerobics } & $37.73 \pm 1.92$ & $37.19 \pm 1.89 a$ & $38.50 \pm 2.25 b$ \\
\hline & PT & & \\
\hline & $\begin{array}{l}\text { Before the } \\
\text { test }\end{array}$ & Immediately & $\begin{array}{l}1 \mathrm{~h} \text { after the } \\
\text { test }\end{array}$ \\
\hline $\begin{array}{l}\text { resistance } \\
\text { training }\end{array}$ & $13.60 \pm 0.13$ & $13.16 \pm 0.16 c$ & $13.81 \pm 0.19 d$ \\
\hline Aerobics & $13.62 \pm 0.16$ & $13.20 \pm 0,17 c$ & $13.84 \pm 0,24$ \\
\hline
\end{tabular}

Note: a - meaningful decrease in comparison with basic level; $b$ meaningful increase in comparison with basic level; c - meaningful decrease in comparison with basic level; $d$ meaningful increase in comparison with basic level.

\section{Discussion}

The results showed a 65-75 percent Maximum training session acute aerobic and resistance exercise to exhaustion and oxygen consumption in three sets, 5 to 7 reps with $80 \%$ of one repetition maximum (1RM) both led to the shortening PT time can be significant. But the recovery took off of PT only resistance training groups significantly decreased compared to the initial levels. While in the air training group did not change significantly. Rezaeian et al [9] showed that in both active and inactive groups, PT time of eight minutes of active recovery (pedal at time zero and an arbitrary distance). After submaximal aerobic training with ergometer (70\% maximum heart rate) did not change significantly [9]. Boldt et al. [10] demonstrated that the immersion in cold water PT time was significantly decreased after the warm water and the core body temperature in $39^{\circ} \mathrm{C}$ there was no change in the PT time. In the 45 minute of recovery (out of water) and reducing the temperature, the 
PT in comparison with the control group (early levels before immersion in water) PT 3\% decline. The PT time after moderate activity by $80 \%$ in young men and older individual anaerobic threshold did not change [10]. While Hilberg et al [11] showed up after a test phase, the PT in healthy subjects increased. Moreover, an increase in PT patients with high blood pressure for 45 minutes on the bike ergometer submaximal exercise was observed that the results have been inconsistent. According Piccione PT responds differently to exercise significant influence represents a type of training with age, sex and condition of the subjects on the coagulation system response [12]. The type of training methods (eg running on the treadmill, pedaling on a bicycle ergometer) intensity exercise can also be involved in these disputes. PT index extrinsic pathway of blood coagulation, and while it depends on the concentration Prothrombin. Since the change in profile was similar in both groups, it can be suggested that the effect of exercise on coagulation pathway activity independent of it is foreign.

The results showed that the aerobic or anaerobic exercise have the same effect on the aPTT time and both of them led to a significant decrease in aPTT volatile and rise above the level of the ground state, is in a recovery. Hilberg et al [13] showed aPTT time to jump from a height of $55 \mathrm{~cm}$ deep extroverted than 90 minutes of exercise at $90 \%$ lactate threshold is much less reduced on a stationary bicycle pedal stronger stimulus to increase Thrombin is potential. Menzel and Hilberg [5] aPTT significant shortening of average activity with $80 \%$ of the anaerobic threshold in young men and the elderly were fixed. APTT decrease in other studies with different protocols running training is evident $[5,6,9,11]$. All of which are consistent with the results of this study show Reduce the time aPTT is not affected by the type of training. aPPP index is much slower than the PT coagulation pathway and mechanism of injury to the blood vessel wall and hit back with collagen begins.

The results show that the intensity and duration of exercise leads to the induction of factor VIII are different. It is also a positive relationship between intensity aerobic

\section{References}

1. El-Sayed MS, El-Sayed Z, Aahmadizad A. Exercise and Training Effects o blood Heamostasis in Health and Disease. Sport Med, 2004;34(3): 181-200. https://doi.org/10.2165/00007256-200434030-00004

2. Wang JS. Exercise prescription and thronbogenesis. Biochem Sci, 2006;13(2): 753-751. https://doi.org/10.1007/s11373-006-9105-7

3. Lippi G, Maffulli N. Biological influence of physical exercise on hemostasis. Semin Thromb Hemost, 2009;35(3): 269-76. https://doi.org/10.1055/s-0029-1222605

4. Habibi M, Torkaman G, Goosheh B, Hedayati M. The effect of combined resistance-aerobic and aerobic training on the coagulative factors of young healthy men. Physiol Pharmacol. 2009; 13 (1):98-107.

5. Menzel,K,HilbergT.Coagulationandfibrinolysisareinbalance after moderate exercise in middle-aged participants. Clinical and Applied Thrombosis/Hemostasis, 2009;15(3):348-355. activity and factor VIII activity and antigen was observed. Similar to aerobic training, resistance training also increases the activity of this factor followed a positive relationship with the volume up resistance [1], on the other hand Lekakis et al. [6] inverse correlation with levels of factor VIII aPTT immediately $(\mathrm{r}=-0.52)$ and one hour after exercise $(\mathrm{r}=-0.75)$ in patients with hypertension Showed. The potential increase in the production of thrombin Willebrand factor and factor VIII is a reflection of increased activity. It seems activate the beta-adrenergic pathway important sign of increased heart rate with exercise, it is likely to increase as a mediator in the VIII of practice involved [1]. The results show that the synthesis of nitric oxide block (with nitro-L-arginine methyl ester) Increase Phone-Willebrand factor and factor VIII activity decreased beta-adrenergic receptor stimulation on nitric oxide production, which refers to its role in the coagulation pathway [14].

In this study, we tried as much as possible net effect of both aerobic and anaerobic exercise on the coagulation system anthropometric factors and thus determine the initial state of the control subjects. Especially resistance training plan that is similar to the model used to develop power and muscle is Hypertrophy [15].

\section{Conclusion}

According to the study a submaximal aerobic and resistance training led to moderate and transient in shortening clotting time in the PT, aPTT and the blood coagulation and homeostasis activity in young women is activated and the type of training the same effect on the short profile of routes external and internal endogenous activator of prothrombin blood clotting and building complex. Workouts with a study leading to a significant risk of thrombosis in patients Substantial not activated, but the implementation of this type of training, further investigation is needed by patients and the elderly.

\section{Conflict of interest}

The authors declare no conflict of interest.

https://doi.org/10.1177/1076029608326306

6. Lekakis J, Triantafyllidi H, Galea V. The immediate effect of aerobic exercise on haemostatic parameters in patients with recently diagnosed mild to moderate essential hypertension. G Thromb Thrombolysis, 2008;25(2): 179-84. https://doi.org/10.1007/s11239-007-0058-0

7. Hilberg T, Glaser D, Reskhart C, Prasa D, Sturzebecher J, Gabriel HH. Blood coagulation and fibrinolysis after long-duration treadmill exercise controlled by individual anaerobic threshold. Eur J Appl Physiol, 2003;90: 639-642. https://doi.org/10.1007/s00421-003-0907-2

8. Nagelkirk PR, Scalzo R, Harber M, Kaminsky LA. The influence of acute Resistance Training and body composition on coagulation and fibrinolytic activity in low risk women. Int G spoets med, 2010;31(7): 458-462. https://doi.org/10.1055/s-0030-1249623

9. Rezaiean Z, Torkaman G, Nadali F. Effect of physical fitness on the coagulate activity of healthy 
young men. Pak J Biol Sci, 2006;9(11): 2032-203. https://doi.org/10.3923/pjbs.2006.2032.2039

10.Boldt LH, Fraszl W, Röcker L, Schefold JC, Steinach M, Noack T, Gunga HC. Changes in the haemostatic system after thermoneutral and hyperthermic water immersion. European journal of applied physiology, 2008;102(5): 547-554. https://doi.org/10.1007/s00421-007-0620-7

11.Hilberg T, Eichler E, Glaser D. Blood coagulation and fibrinolysis before and after exhaustive exercise in patients with IDDM. Thromb Haemosd, 2003;90(6): 1065-73.

12.Picciome Fazio GF, Giudice E. Exercise induced change in clotting times and fibrinolytic activity during official 1600 and 2000 meters' trot races in standard bred horses. Acta Vet Brno, 2005;74(3): 509-514. https://doi.org/10.2754/avb200574040509
13.Hilberg TG, Glaser D, Prasa D. Pure eccentric exercise does not activate blood coagulation. European journal of applied physiology, 2005;94(5-6): 718-721. https://doi.org/10.1007/s00421-005-1353-0

14. Madarame H, Kurano M, Takano H, Lida H, Sato Y, Ohshima $\mathrm{H}$. Effect of low-intensity resistance exercise with blood flow restriction on coagulation system in healthy subjects. Clinical physiology and functional imaging, 2010;30(3): 210-213. https://doi.org/10.1111/j.1475-097X.2010.00927.x

15.Ahmadizad S, El-Sayed M. The acute effects of resistance exercise on the main determinants of blood rheology. Journal of Sports sciences, 2005;23(3): 243-249. https://doi.org/10.1080/02640410410001730151

\section{Information about the authors:}

Rahmani Ghobadi M.; (Corresponding author); Assistant Professor; http://orcid.org/0000-0002-4948-6895; mrahmani.uni@ gmail.com; Department of Physical Education \& Sport Sciences, Damavand Branch, Islamic Azad University; Moalem Square, Islamic Azad University, Damavand, Tehran Province, Iran.

Forghani Ozrudi M.; http://orcid.org/0000-0003-1683-3106; mohammadbagher.forghani@gmail.com; Young Researchers and Elite Club, Babol Branch, Islamic Azad University; Mazandaran province, Babol City, Education office, Department of physical education, Babol, Iran.

\section{Cite this article as:}

Rahmani Ghobadi M, Forghani Ozrudi M. The comparing of acute effects of two training models aerobic and resistance on the clotting times in young women students. Physical education of students, 2019;23(4):198-201.

https://doi.org/10.15561/20755279.2019.0406

This is an Open Access article distributed under the terms of the Creative Commons Attribution License, which permits unrestricted use, distribution, and reproduction in any medium, provided the original work is properly cited http://creativecommons.org/licenses/by/4.0/deed.en

Received: 20.05.2019

Accepted: 22.06.2019; Published: 28.08.2019 